\title{
Case Report: A Complicated Maternal Death in the Context of Genetic Disorder: A Case Report
}

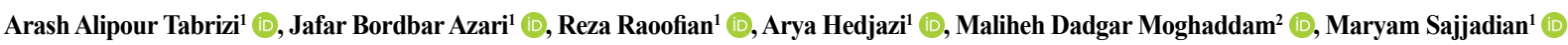

1. Legal Medicine Research Center, Legal Medicine Organization, Tehran, Iran.

2. Department of Community Medicine, School of Medicine, Mashhad University of Medical Science, Mashhad, Iran.

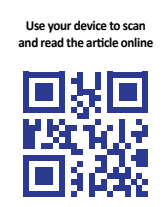

Chtation: Alipourtabrizi A, Bordbar Azari J, Raoofian R, Hedjazi A, Dadgarmoghaddam M, Sajjadian M. A Complicated Maternal Death in the context of Genetic Disorder: A Case Report. International Journal of Medical Toxicology and Forensic Medicine. 2021; 11(1):32308. https://doi.org/10.32598/ijmtfm.v11i.32308

doi https://doi.org/10.32598/ijmtfm.v11i.32308

\section{(c) (1) (\$)}

Article info:

Received: 20 Sep 2020

First Revision: 27 Sep 2020

Accepted: 17 Oct 2020

Published: 30 Mar 2021

\section{Keywords:}

Neurofibromatosis type 1, Pheochromocytoma, Maternal death, Hypertension, Pulmonary edema

\section{ABSTRACT}

Background: Investigation of the maternal cause of death and pregnancy-related death is one of the most important responsibilities of a forensic pathologist. From the public health point of view, it may help to prevent losses during and following pregnancy and save the lives of women, especially in developing countries.

Methods: We report a case of maternal death with a history of neurofibromatosis type 1 who presented asymptomatic and normotensive with normal laboratory test results.

Results: The first attack after delivery was associated with pulmonary edema, which led to death. Investigation during the medico-legal autopsy discovered a left suprarenal tumor with the diagnosis of pheochromocytoma, which was confirmed by further histopathology testing.

Conclusion: We believe that although the association of neurofibromatosis type 1 and normotensive pheochromocytoma during pregnancy has been reported rarely, the possibility must be considered for evaluation before elective operations to adopt proper preoperative protocols. 


\section{Introduction}

aternal death occurs exclusively in women and is an indicator of health$\mathbf{M}$ related Sustainable Development Goals (SDGs). This preventable tragedy is concentrated in low-income countries as one woman out of 41 dies from maternal causes. Therefore, the prevention of maternal deaths is one of the main goals in many developing countries [1]. A case of maternal death referred to the Legal Medicine Organization occurred just after $14 \mathrm{~h}$ of admission to the hospital without any diagnosis for the cause of death.

\section{Case Report}

A 35 years old Iranian housewife with a history of second Gravidity and one Parity (G2P1) was under prenatal care of a gynecologist. This second wanted pregnancy was discovered along with an indistinctive last menstrual period after four years of contraception by Oral Contraceptives (OCPs).

In the seventeen weeks of gestational age, the case was filed at the urban health center for routine medical examinations and prenatal screening tests leading to normal lab results. No clear history of chronic illness, hematemesis, and hypertension was expressed by the patient.

Considering the physical examinations, family history, and her medical records, the woman was a proven case of the neurocutaneous genetic disorder, Neurofibromatosis type 1 (NF1), or von Recklinghausen's disease from the paternal origin. The hallmarks of NF1, the multiple café-au-lait spots, and associated cutaneous neurofibromas were seen in the patient (Figure 1).

On the morning of the midday of 39 gestational weeks, she was admitted to the Department of Obstetrics and Gynecology for cesarean section and tubal ligation procedures because of the previous classical cesarean section. Normal vital signs were registered (pulse rate $=82 \mathrm{~min}$, respiratory rate $=18 \mathrm{~min}$, blood pressure $=100 / 60 \mathrm{mmHg}$, body temperature $=37^{\circ} \mathrm{C}$, and fetal heart rate $=135 \mathrm{~min}$ ).

A history of penicillin allergy was mentioned by the patient and considered for antibiotic therapy. After the operation, a normal newborn baby with an Apgar score of 9 was delivered. No anesthetic and hemostatic issues occurred and the patient was referred to the recovery room with a good general condition.
Symptomatic episodic tachycardia was detected (heart rate $=155 \mathrm{~min}$ ) $7 \mathrm{~h}$ post-operation and continued by pink vomiting and hemoptysis, complicated by tachypnea and clear pulmonary rales, $2 \mathrm{~h}$ later.

Due to the diagnosis of acute pulmonary edema and the supposition of pulmonary embolism, the patient was referred to the Intensive Care Unit (ICU), and Cardiopulmonary Resuscitation (CPR) was performed immediately. The patient died after $2 \mathrm{~h}$ and the body was transferred to the Legal Medicine Center of Mashhad for medical autopsy.

Autopsy findings showed pulmonary edema without any noticeable signs of pulmonary embolism or main vascular embolisms. An abdominal round mass of $4 \mathrm{~cm}$ in diameter, in the left suprarenal area, close to the spleen, was resected for further microscopic investigations.

Microscopic examination showed severe pulmonary edema without any significant pathological changes in the kidney, liver, heart, brain, and uterus tissue samples. Mass resection specimen grossly revealed a firm deep brown mass. Cut sections showed areas of central hemorrhage (Figure 2). The microscopic examination also showed the tumoral arrangement in multiple cellular nests (Zellballen Pattern) within the fibrovascular stroma. Round nucleus, some with prominent nucleoli and granular cytoplasm, and focal hyaline globules were seen microscopically (Figure 3).

Immunohistochemical staining with chromogranin showed positive tumoral cells with diffuse cytoplasmic staining. Immunostaining for S100 protein showed a discontinuous layer of sustentacular cells around the cell nests (Figure 4). All the data confirmed the pheochromocytoma as the final diagnosis and possible cause of death.

\section{Discussion}

We reported a case of unilateral pheochromocytoma with a history of NF1 without a history of sustained or paroxysmal hypertension. The prevalence of NF1 is about 1 in 3000 live births worldwide and patients with NF1 have a higher chance to develop some special tumors, including pheochromocytoma [2].

The pheochromocytoma originates from medullary parts of the adrenal glands; therefore, it can cause secondary hypertension, and pheochromocytomas are rarely malignant. Although most pheochromocytomas are in a sporadic form, familial types are seen in cases of Multiple Endocrine Neoplasia 2 (MEN2), NF1, and Von 


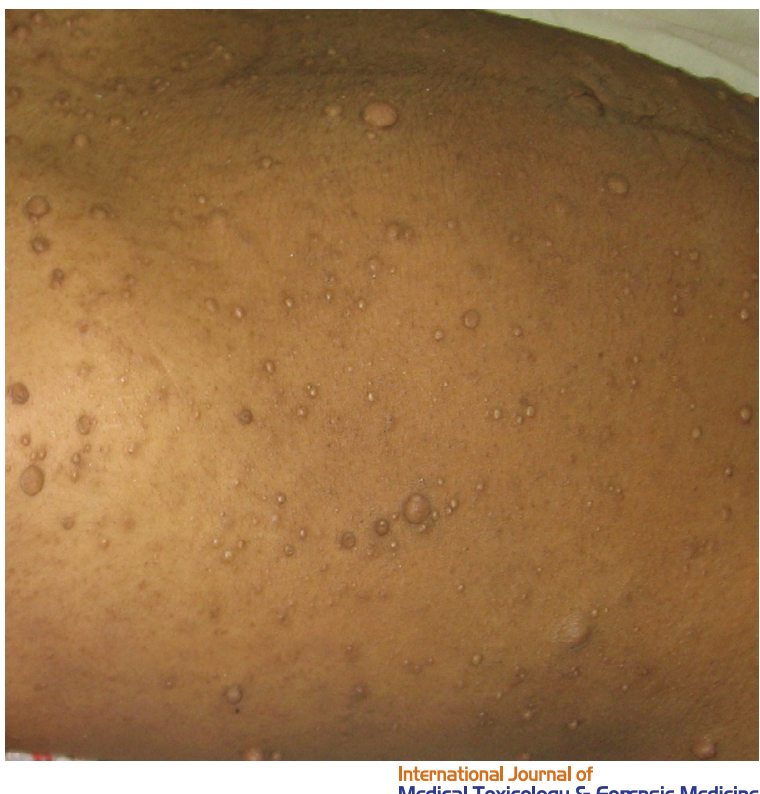

Medical Toxicology \& forensic Medicine Figure 1. Multiple neurofibromas and a Café au lait spot located at the left of the thorax

Hippel-Lindau (VHL) disease as an autosomal dominant inherited trait with complete penetrance. The rate of pheochromocytoma in NF1 patients is $0.1 \%$ to $5.7 \%$ in different populations, but it rises to $20 \%-50 \%$ in hypertensive NF1 patients.

The classic triad of episodic headache, sweating, and palpitations caused by catecholamine's release from the tumor may lead to a hypertensive crisis, arrhythmia, or myocar-

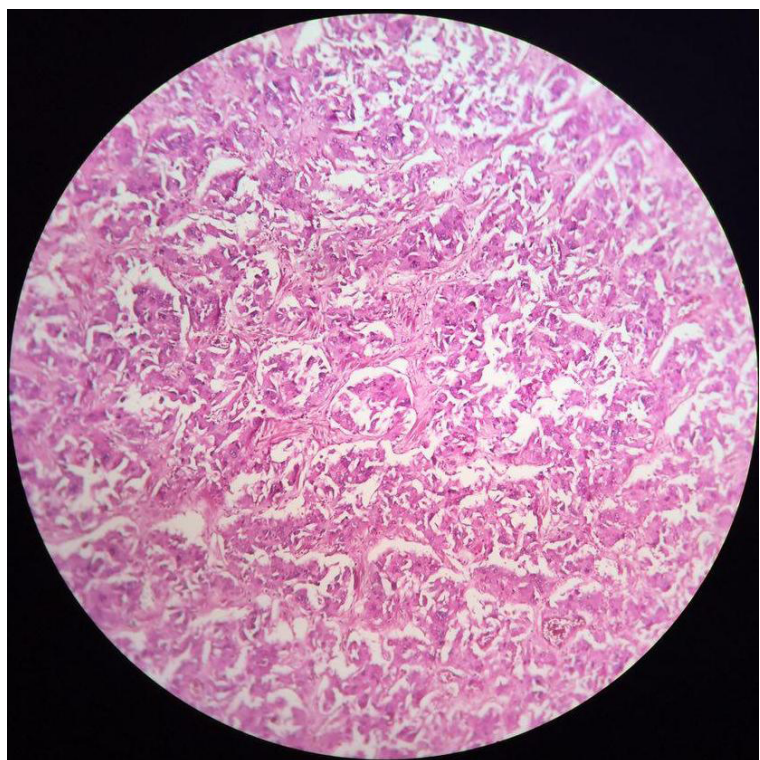

Figure 3. Histopathological examination

A: Normal adrenocortical tissue close to the tumoral mass; B: Well-defined nests of tumor cells (zellballen) with granular cytoplasm and round nuclei, separated by highly vascularized fibrous septa (H\&E×100).

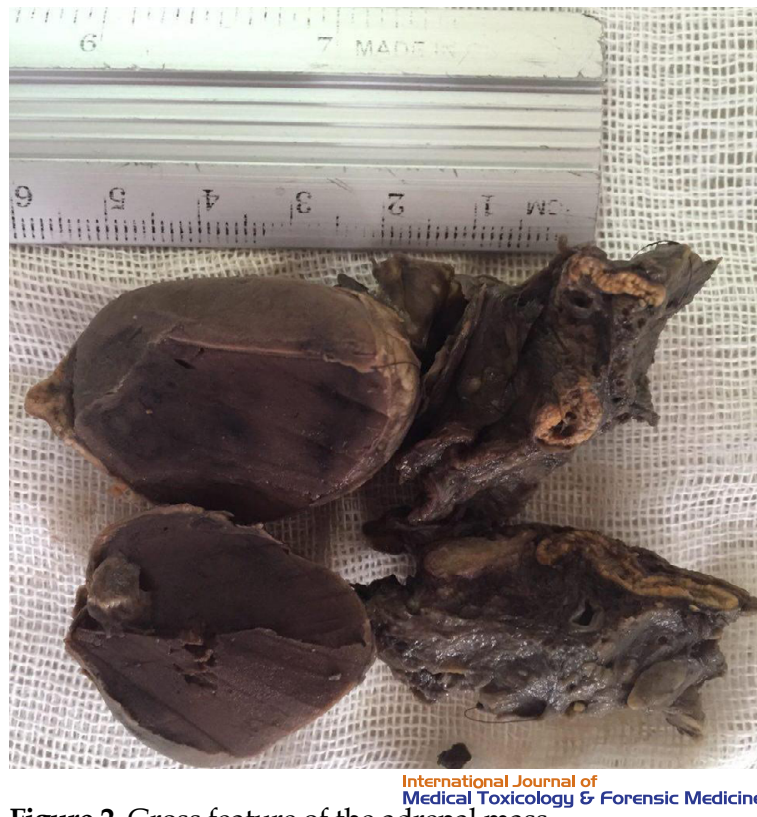

Figure 2. Gross feature of the adrenal mass

dial infarction, and death in undiagnosed pheochromocytoma cases, which are mainly hypertensive patients [3]

The prevalence of pheochromocytoma is nearly $0.2 \%$ in autopsy series and myocardial infarction and cerebrovascular catastrophes are the cause of death in $75 \%$ of pheochromocytomas found in postmortem investigations $[4,5]$.

Hypertension due to pheochromocytoma may be challenging because it can mimic pregnancy-induced hy-

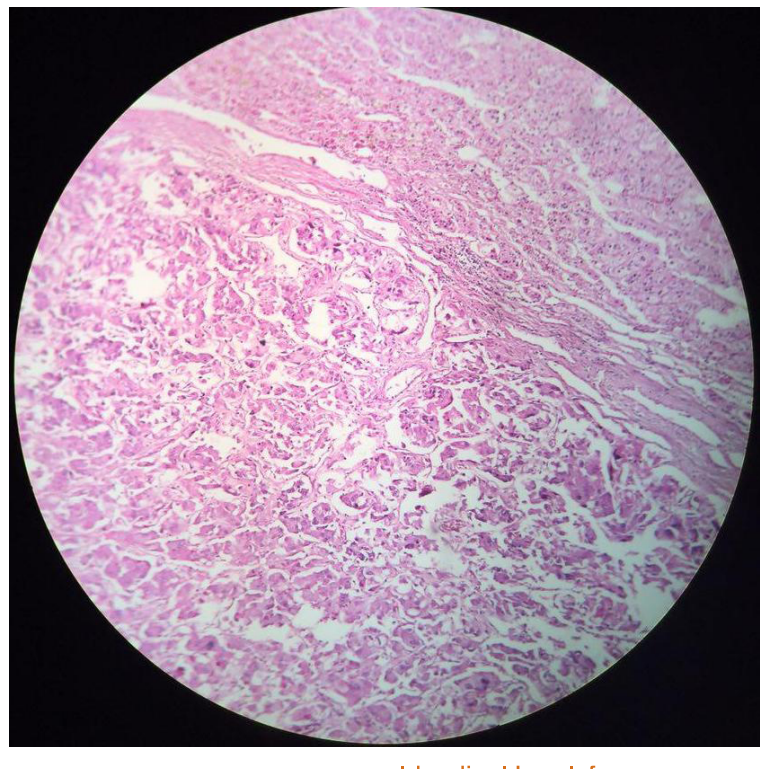

International Journal of 


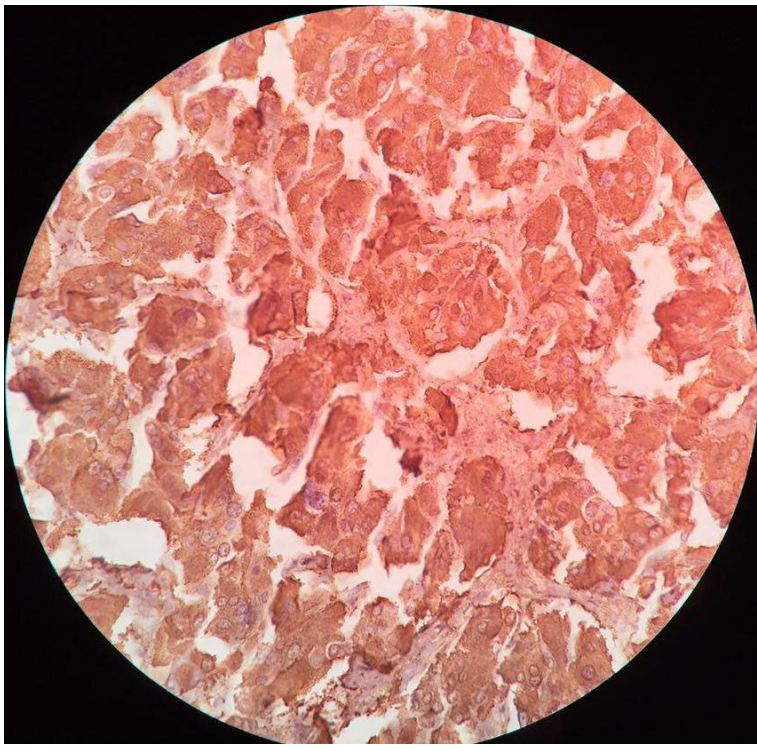

Figure 4. Immunohistochemical staining

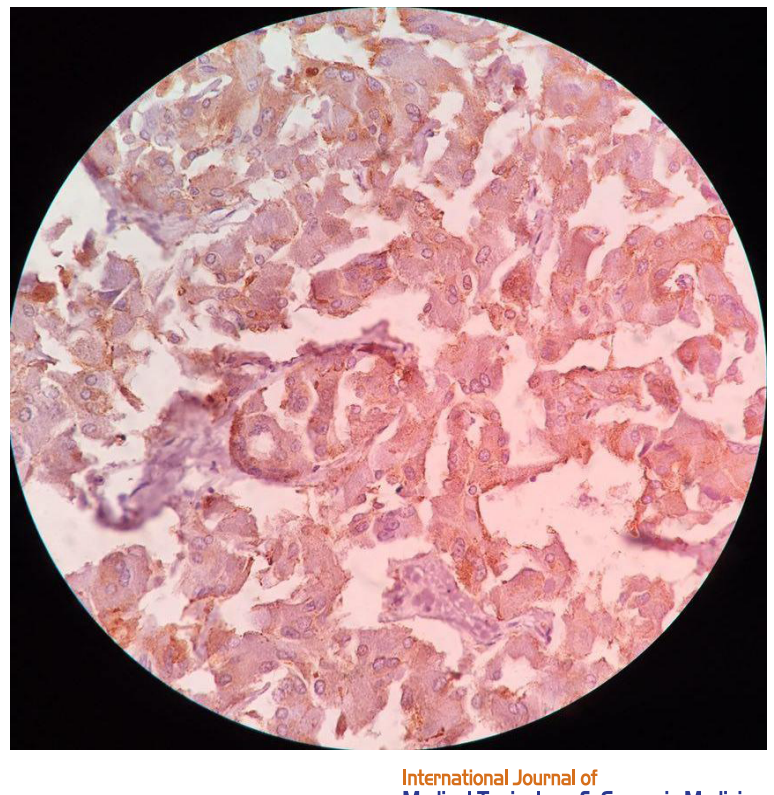

Medical Toxicology \& Forensic Medicine

A. Immunoreactivity for chromogranin: cytoplasmic staining in the tumor cells (IHC $\times 100)$; B. Highlighted sustentacular cells are immunostained for S100 protein, supporting the tumor nests (IHC $\times 100)$.

pertension (preeclampsia, gestational hypertension, and essential hypertension) and must be differentiated from other causes of cardiovascular collapse, such as pulmonary embolisms or amniotic fluid embolisms. The fatal manifestation of pheochromocytoma, in this case, was pulmonary edema, which is uncommon.

Severe hypertension due to pheochromocytoma can cause cardiogenic pulmonary edema, but non-cardiogenic pulmonary edema was found due to increased pulmonary capillary pressure and capillary permeability, secondary to the catecholamine release, as well $[6,7]$.

Based on the literature, nearly $10 \%$ of pheochromocytomas are found in normotensive patients. It is argued that in normotensive patients, pheochromocytoma secrets ten substances, like dopamine along with or in the absence of other catecholamines, which caused patients to be normotensive, but this clinically silent pheochromocytoma can be lethal during or after operative procedures [8].

The first report of NF1 associated with pheochromocytoma was described by Suzuki in 1910 [9]. Although the incidence of this association is mentioned very low, the correct diagnosis of pheochromocytoma in patients with NF1 history allows the right treatment and preoperative preparation with adrenergic blockers.

Albeit, adoption of the routine screening strategy for pheochromocytoma in all NF1 patients may be accepted after evaluation of cost-effectiveness, but our case high- lights the need for evaluating silent pheochromocytoma in NF1 patients admitted for elective surgical operations to prevent the morbidity and mortalities not only for hypertensive but also in normotensive ones.

\section{Conclusion}

In conclusion, although the coexistence of pheochromocytoma with NF1 during pregnancy, with a normotensive manifestation history, is extremely rare, preoperative evaluations should be considered as a preventive strategy for further forensic issues.

\section{Ethical Considerations}

\section{Compliance with ethical guidelines}

All ethical principles are considered in this article. The participants were informed about the purpose of the research and its implementation stages. They were also assured about the confidentiality of their information and were free to leave the study whenever they wished, and if desired, the research results would be available to them.

\section{Funding}

This research did not receive any grant from funding agencies in the public, commercial, or non-profit sectors. 


\section{Author's contributions}

Conceptualization: Arash Alipourtabrizi, Jafar Bordbar Azari, and Maryam Sajjadian; Investigation: Reza Raoofian and Jafar Bordbar Azari; Writing - original draft: Arash Alipourtabrizi; Writing - review \& editing: Maliheh Dadgarmoghaddam; Supervision: Arya Hedjazi.

\section{Conflict of interest}

The authors declared no conflict of interest.

\section{References}

[1] World Health Organization (WHO). World health statistics overview 2019: Monitoring health for the SDGs, sustainable development goals. Geneva: World Health Organization; 2019. https://apps.who.int/iris/handle/10665/324835. License: CC BY-NC-SA 3.0 IGO

[2] Rocchietti March M. Type 1 neurofibromatosis and pheochromocytoma: Focus on hypertension. J Neurosci Rural Pract. 2012; 3(1):107-8. [DOI:10.4103/0976-3147.91987] [PMID] [PMCID]

[3] Dluhy RG. Pheochromocytoma: Death of an axiom. N Engl J Med. 2002; 346(19):1486-8. [DOI:10.1056/ NEJM200205093461911] [PMID]

[4] Candanedo-Gonzalez F, Camacho-Rebollar L, Cordova-Uscanga C. Macro and microscopic aspects. Pheochromocytoma - A New View of the Old Problem. 2011; 1-14. [DOI: 10.5772/25426]

[5] Longo D, Fauci A, Kasper D, Hauser S, Jameson J, Loscalzo J. Harrison's principle of internal medicine: Volumes 1 and 2, 18th Edition. Pennsylvania Plaza, New York City: McGrawHill Professional Pub; 2011. https://books.google.com/ books?id=7gxjMV8hClsC\&q

[6] Haddad S, Al-Raiy B, Madkhali A, Al-Qahtani S, Al-Sultan $\mathrm{M}$, Arabi Y. Cardiorespiratory crisis at the end of pregnancy: A case of pheochromocytoma. Middle East J Anaesthesiol. 2013; 22(2):195-202. [PMID]

[7] Plu I, Sec I, Barrès D, Lecomte D. Pregnancy, cesarean, and pheochromocytoma: A case report and literature review. J Forensic Sci. 2013; 58(4):1075-9. [DOI:10.1111/1556-4029.12107] [PMID]

[8] Nazir O, Sharma T, Maqsood M, Khatuja A, Misra R. Epinephrine-secreting large incidental pheochromocytoma in a normotensive male with stormy intraoperative hemodynamics. Gulf J Oncolog. 2015; 1(18):13-7. [PMID]

[9] Suzuki S. [About two tumors from adrenal medulla tissue (Ueber zwei tumoren aus nebennierenmarkgewebe) (German)]. Berlin Klein Wchnschr. 1910; 47:1623. 